\title{
Métodos físicos e químicos de detoxificação de aflatoxinas e redução da contaminação fúngica na cadeia produtiva avícola
}

\author{
Physical and chemical methods of detoxification of aflatoxins and reduction of fungal contamina- \\ tion on poultry productive chain
}

\author{
Ágatha Cristina de Pinho Carão ${ }^{I^{*}}$ Maria Fernanda de Castro Burbarelli ${ }^{\mathrm{I}}$ \\ Gustavo do Valle Polycarpo ${ }^{I}$ Amanda Ramos dos Santos $^{\mathrm{I}}$ Ricardo de Albuquerque $^{\mathrm{I}}$ \\ Carlos Augusto Fernandes de Oliveira ${ }^{I I}$
}

\section{RESUMO}

\begin{abstract}
As aflatoxinas são responsáveis por grandes prejuízos à saúde de aves comerciais e à produção animal, essencialmente por perda financeira decorrente de diminuição de ganho de peso dos animais. A detoxificação da ração ou de suas matérias-primas, bem como tentativas de diminuir a contaminação fúngica que leva à produção das toxinas, além de técnicas nutricionais, são métodos que podem ser adotados para tentar diminuir os efeitos deletérios sobre os frangos de corte. Sendo assim, o objetivo desta revisão é mostrar o que a ciência fornece como meios de detoxificação e/ou redução de contaminação por fungos; $e$ algumas substâncias naturais que auxiliam a produção avícola na redução de micotoxicoses .
\end{abstract}

Palavras-chave: avicultura, contaminação fúngica, micotoxinas, detoxificação.

\section{ABSTRACT}

Aflatoxins are answerable for large losses for the health of commercial birds and for the animal production, essentially by financial losses due to animal body weight diminution. Detoxification of feed or its raw materials, well as attempts of diminution of fungal contamination that leads to toxins production, beyond of nutritional techniques, are methods that can be adopted trying to diminish deleterious effects among broiler chickens. This way, the objective of this review is to show what science supplies like ways of detoxification and/or reduction of contamination by fungi, and some natural substances that help poultry production on reduce mycotoxicosis.

Key-words: poultry production, fungal contamination, mycotoxin, detoxification.

\section{INTRODUÇÃO}

Micotoxinas são metabólitos secundários produzidos por fungos que podem entrar na dieta de seres humanos e animais por meio de contaminação direta ou indireta de cereais e grãos. São difíceis de diagnosticar e, de acordo com suas propriedades físico-químicas e a espécie animal envolvida, cada micotoxina pode afetar especificamente um órgão ou sistema, levando a manifestações clínicas específicas de natureza aguda ou crônica (PIER. et al., 1973).

Aflatoxicoses são micotoxicoses causadas por toxinas classificadas como aflatoxinas, produzidas por cepas de fungos do gênero Aspergillus, (A. flavus, $A$. parasiticus e $A$. nominus) descobertas em 1960, após provocarem um surto tóxico em perus na Inglaterra (Turkey-X-disease) em que milhares de aves morreram após consumirem torta de amendoim na ração, proveniente do Brasil, e as aves doentes apresentaram necrose do tecido hepático (LEESON et al., 1995). Os principais compostos de interesse médico-sanitário são identificados como B1, G1, B2 e G2; sendo que a aflatoxina B1 (AFB1), além de ser a mais frequentemente encontrada em cereais, é que apresenta maior poder toxigênico (LEESON et al., 1995). A toxicidade das aflatoxinas depende de sua concentração, sua duração da exposição, a espécie, o sexo, a idade e a condição de saúde dos

\footnotetext{
'Departamento de Nutrição e Produção Animal, Faculdade de Medicina Veterinária e Zootecnia, Universidade de São Paulo (USP), Pirassununga, SP, Brasil. E-mail: agathapinho@usp.br. *Autor para correspondência.

${ }^{I I}$ Departamento de Engenharia de Alimentos, Faculdade de Zootecnia e Engenharia de Alimentos, USP, 13635-900, Pirassununga, SP, Brasil. 
animais (JEWERS, 1990). Na aflatoxicose crônica, o sinal clínico mais evidente é a diminuição da taxa de crescimento de animais jovens (LEESON et al., 1995). A síndrome tóxica aguda ocorre pela ingestão de alimento com alta concentração de aflatoxina, sendo os efeitos observados em curto espaço de tempo. As aflatoxinas induzem a vários efeitos biológicos, tais como doenças hepáticas, alterações na taxa de crescimento, mudanças nos mecanismos imunogênicos, e efeitos carcinogênicos e mutagênicos em espécies animais diferentes, especialmente em aves domésticas (PIER et al., 1973).

Métodos de combate às aflatoxinas

Visando a diminuição dos efeitos deletérios causados pelas aflatoxinas, há, basicamente, três técnicas possíveis para evitá-los: a prevenção e/ ou a diminuição da contaminação do alimento, a detoxificação do alimento e o impedimento da absorção das micotoxinas pelo trato digestivo dos animais. Porém, qualquer que seja a estratégia de descontaminação usada, ela deve ir de encontro a alguns critérios básicos (PARK \& POMERANZ et al. apud BATA \& LÁSZTITY, 1999): a micotoxina deve ser inativada (destruída) pela transformação em compostos não-tóxicos; os esporos e o micélio de fungos devem ser destruídos, afim de que novas toxinas não sejam formadas; o material do alimento ou da ração deve reter seu valor nutritivo e permanecer palatável; as propriedades físicas do material cru não devem ser alteradas significativamente, e deve ser economicamente viável.

Inativação física

Enquadra-se nos meios de detoxificação dos alimentos já contaminados.

Extração por solventes

Alimentos tratados por este método só podem ser utilizados para a alimentação animal. Os solventes usados incluem: 95\% de etanol, 90\% de acetona aquosa, 80\% de isopropanol, hexanometanol, metanol-água, acetonitrila-água, hexanoetanol-água e acetona-hexano-água. A extração por solventes pode remover todos os traços de aflatoxinas das farinhas de sementes oleaginosas sem a formação de sub-produtos tóxicos ou redução na qualidade ou no conteúdo de proteína. Entretanto, esta técnica em larga escala limita-se por altos custos e problemas relacionados à disposição dos extratos tóxicos (RUSTOM, 1997).

Tratamento térmico (Aquecimento, Extrusão e Tratamento por Microondas)
O tratamento térmico utilizado é com auxílio de calor. A extensão da destruição alcançada é muito dependente de: nível de contaminação inicial, temperatura de aquecimento, tempo de exposição ao calor, tipo de alimento e de aflatoxina, além de umidade, $\mathrm{pH}$ e concentração iônica do alimento. $\mathrm{O}$ conteúdo de umidade é um fator crítico; alimentos contaminados que possuem maior umidade podem ser mais facilmente inativados pelo calor (RUSTOM, 1997). De acordo com HWANG \& LEE (2006), o tratamento térmico é capaz de produzir reduções significativas de contaminação de aflatoxina B1 em aveia não-umedecida ( $>80 \%$ de umidade, a temperatura de $200^{\circ} \mathrm{C}$, durante 30,60 ou 90 minutos $)(\mathrm{P}<0,01)$.

A extrusão é um tipo de tratamento térmico de que a indústria se utiliza para a produção de muitos alimentos. Para destruir ou inativar aflatoxinas, as condições de cozimento da extrusão precisam ser severas (alto cisalhamento, alta temperatura e correto $\mathrm{pH}$ ) afim de providenciar o ambiente necessário no tambor. SAALIA \& PHILLIPS (2011), trabalhando com farinha de amendoins naturalmente contaminados por aflatoxinas $\left(200 \mu \mathrm{g} \mathrm{kg}^{-1}\right.$ de B1 e G1 e $60 \mu \mathrm{g} \mathrm{kg}^{-1}$ de B2 e G2) e submetendo-a a extrusão com 35\% de umidade, obtiveram redução de $59 \%$ na quantidade de aflatoxinas detectáveis por HPLC (Cromatografia Líquida de Alta Performance), sem prejuízo ao valor nutricional protéico do alimento. H A M E E D (1993) trabalhando com extrusão de farinha de milho contaminada com 500ppb de aflatoxinas, obteve redução de $70 \%$ nos níveis de contaminação com auxílio somente do processo de extrusão.

$\mathrm{O}$ aquecimento por microondas é outra técnica que pode ser aplicada com mesmo objetivo. BASARAN \& ÜMRAN (2010) afirmam haver redução da contaminação de nozes por $\boldsymbol{A}$. parasiticus (o que contribuiria para a redução da concentração de aflatoxinas ao longo do período de estocagem) em dois logaritmos após a exposição da superfície das nozes a microondas por 60 segundos, sob temperatura externa da casca em $50-55^{\circ} \mathrm{C}$. Após 120 segundos de exposição, até 2,5 logarítmos de redução foi possível. Quando testou-se a efetividade das microondas em diminuir a concentração das aflatoxinas já presentes nas nozes, estas foram ineficazes. Os níveis de aflatoxinas permaneceram inalterados $(\mathrm{P}>0,05)$ sob $2,45 \mathrm{GHz}$ por 120 segundos.

Irradiação (Irradiação Solar, Raios Gama e Luz Ultravioleta)

A técnica mais simples consiste na secagem de materiais sob exposição à luz solar. GOWDA et al. (2007) constataram redução de 83,7\% (de 326 
para $52 \mathrm{ppb}$ de aflatoxina B1) em dieta de ovelhas contaminada artificialmente e exposta à luz solar por 14 horas, em temperaturas entre 27 e $35^{\circ} \mathrm{C}$.

A irradiação de alimentos é um processo físico que envolve a exposição do alimento a um dos três tipos de energia ionizante: radiação gama, raios-X ou feixe de elétrons. A mais usada para processamento de alimentos é a de radiação gama, proveniente do radioisótopo Cobalto-60 (DULLEY apud CAMARGO et al., 2011). Existem trabalhos que sugerem que os fungos são muito sensíveis à radiação gama, e em adição, a sua produção de micotoxina diminui após a irradiação (REFAI et al. e YOUSSEF et al., apud AQUINO et al., 2005). AQUINO et al. (2005), avaliando o efeito da radiação gama sob a sobrevivência dos fungos da espécie $\boldsymbol{A}$. flavus e a destruição das aflatoxinas B1 e B2, afirmam haver redução do número de unidades formadoras de colônia do fungo $(\mathrm{P}<0,01)$ e a destruição parcial das toxinas sob 2 e $5 \mathrm{kGy}$ de irradiação, bem como a destruição total das toxinas sob $10 \mathrm{kGy}$ de irradiação em milho artificialmente contaminado (Umidade relativa de $97-99 \%$ e atividade de água de $0,88-0,94)$. SIMAS et al. (2010) também relatam haver melhora nos parâmetros de produção zootécnica de frangos alimentados com dieta submetida a radiação gama de $5 \mathrm{kGy}$.

A radiação ultravioleta (UV) também pode ser aplicada. Ela tem efeito microbicida se utilizada com intensidade e tempo de exposição suficientes. As irradiações ultravioletas na faixa de 210 e 330nm são mais eficientes como germicidas por serem absorvidas pelas proteínas e ácidos nucléicos, provocando o rompimento de cromossomo, mutações genéticas e inativação de enzimas e, consequentemente a morte da célula (CARDOZO apud ALEXANDRE et al., 2008). O método mais prático de gerar radiação UV é aquele das lâmpadas germicidas (ALEXANDRE et al., 2008). SAMARJEEWA et al. (1990) afirmam que a radiação gama pode reduzir de 75 a $100 \%$ os níveis de aflatoxina na farinha de amendoim; que a luz ultravioleta reduz até $50 \%$ a concentração de aflatoxina; bem como a irradiação solar de 50 a $90 \%$ da toxina presente.

Inativação química

Métodos químicos consistem em degradar ou inativar as micotoxinas, com uso de ácidos, bases, aldeídos, agentes oxidantes e gases (NORRED, 1993). A inativação química das aflatoxinas pode ser realizada com o auxílio de compostos que induzam modificações químicas irreversíveis na molécula e que estas substâncias não sejam tóxicas aos seres vivos. Porém, em sua grande maioria, estes métodos podem ser não-práticos, ineficazes ou inseguros, além de diminuir os níveis nutricionais e o paladar do alimento.

\section{Amonização}

O tratamento com amônia em forma de gás, em solução ou com substâncias capazes de liberá-la, alcançou resultados ótimos na detoxificação de farelo de amendoim, milho e algodão (PIVA et al., 1995). SAMARJEEWA et al. (1990), trabalhando com concentrações que variavam de 0,5 a $5,0 \%$ de amônia sobre diferentes substratos mostram haver redução acima de 93\% nos níveis de contaminação por aflatoxina. THANABORIPAT et al. (1992) também testaram a ação do hidróxido de amônia sobre milho contaminado experimentalmente por A. flavus produtores de AFB1 e AFG1. Quando adicionados $0,2,0,5,1,0$ e $1,5 \%$ de $\mathrm{Nh}_{4} \mathrm{OH}$ houve redução de produção de 45 a 100\% de AFB1 + AFG1. Porém, para se trabalhar com amônia, é necessária uma planta especial e cuidado, uma vez que o gás pode sofrer combustão em misturas com ar em volumes acima de $15 \%$, além de alterações nutricionais e organolépticas (PIVA et al., 1995).

\section{Ozonização}

A ozonização é uma técnica que está apresentando uma técnica promissora. O gás ozônio apresenta certas características sanitizantes atraentes para a indústria alimentícia, por ser mais seguro e potente do que os desinfetantes convencionais, agindo sobre um grande número de microorganismos. Age através do ataque direto do ozônio e do ataque através dos radicais $\mathrm{OH}$ formados na decomposição do $\mathrm{O}_{3}$ (GIORDANO, 2009). KELLS et al. (2001) notaram redução de $63 \%$ na contaminação por $A$. parasiticus em milho após exposição a 50ppm de ozônio durante 3 dias. Em seu trabalho, GIORDANO et al. (2012) afirmam haver redução da contaminação fúngica (de 4,83 para $0 \log$ ufc $\mathrm{g}^{-1}$ de A. flavus e A. parasiticus) e não-detecção de aflatoxinas em castanha-do-Pará submetidas a ozonização $\left(14\right.$ e $\left.31,5 \mathrm{mg} \mathrm{l}^{-1}\right)$ por 5 horas. $\mathrm{Na}$ tentativa de provar que o ozônio obtido através de eletrólise é capaz de conter os efeitos negativos da aflatoxina B1 em perús jovens, McKENZIE et al. (1998) forneceram milho contaminado (1220+/$73,3 \mathrm{ppb}$ de AFB1) e tratado com 200mg min-1 de $\mathrm{O}_{3}$ por 92 horas aos animais. Houve redução de $95 \%$ da micotoxina na ração tratada e reteve os efeitos indesejáveis da AFB1 na produção animal.

\section{Acidificação}

Ácidos orgânicos também podem ser usados como químicos fungistáticos, que impedem 
o crescimento da população de fungos e a produção de micotoxinas. O mais eficiente custo-benefício tem sido encontrado com o uso de ácido propiônico (AlHILLI \& SMITH, 1992). Além das concentrações de 500, 1000 e $1500 \mu \mathrm{g} \mathrm{g}^{-1}$ de ácido propiônico não terem sido capazes de inibir a produção de aflatoxinas B1 e G1 por A. flavus, funcionaram como substrato para a síntese microbiana. Ácidos fracos, com baixo potencial de ionização, são potentes inibidores do transporte de aminoácidos por parte da célula fúngica, pela ionização interna do citoplasma e acidificação do conteúdo celular, dificultando a sobrevivência do microorganismo Ledoux (2012 - Informe verbal).

Uso de substâncias naturais

Os extratos herbais vêm sendo estudados com o intuito de uso em alimentos para inibição de crescimento fúngico e consequente produção de toxinas, muito embora atualmente também os empregue como meio nutricional de combate aos efeitos negativos das micotoxicoses. Óleos essenciais extraídos de ervas comestíveis constituem uma alternativa utilizada em estudos para investigação de possível uso em alimentos, a exemplo de óleos de plantas como orégano, cravo, canela, alho e tomilho (SHAPIRA, 2004). CHINAPHUTI \& AUKKASARAKUL (2008) testaram 15 espécies de ervas que são cultivadas na região da Tailândia e afirmam que a grande maioria pode ser usada como inibidor de crescimento de A. flavus. Extrato brutos de Allium sativum, Occimum tenuiflorum e Occimum basillicum possuem a capacidade de inibir a produção de aflatoxinas, bem como o crescimento do fungo. REDDY et al. (2009) também mostram que o extrato de Syzygium aromaticum $\left(5 \mathrm{~g} \mathrm{~kg}^{-1}\right)$ mostram inibição completa do crescimento de A. flavus e produção de aflatoxina B1. Em menor porcentagem (6578\%), Curcuma longa, Allium sativum e Occimum sanctum também inibem efetivamente a proliferação fúngica e a produção de micotoxina $(72,2-85,7 \%)$ em concentração de $5 \mathrm{~g} \mathrm{~kg}^{-1}$. AL-SOHAIBANI et al. (2011) também relatam que extratos de espécies de pimenta usadas como especiarias e como ervas medicinais (Elettaria cardamomum e Syzygium aromaticum) na Índia possuem efeito inibitório e podem ser usados como antimicrobianos em chás. Entretanto, resultados contraditórios têm sido reportados em muitos casos a respeito da atividade de alguns constituintes de plantas.

Meios nutricionais de prevenção dos efeitos negativos das aflatoxicoses também estão sendo estudados como métodos auxiliares ao combate às lesões patológicas nos animais de criação.
A utilização de vitamina E e selênio foi estudada por HE et al. (2013) na tentativa de melhora de performance e redução de alterações patológicas em patos, o que permitiu que os efeitos negativos da aflatoxina B1 sobre o crescimento e a mortalidade fossem parcialmente contidos (50UI de vitamina $\mathrm{E}+$ $0,2 \mathrm{mg} \mathrm{kg}^{-1}$ de selênio) $(\mathrm{P}<0,05)$. CHEN et al. (2013) demonstraram melhora na função imunológica de frangos alimentados com 0,6 e $0,8 \mathrm{mg} \mathrm{kg}^{-1}$ de selênio e intoxicados com $0,3 \mathrm{ppm}$ de alfatoxina B1, com maior concentração de linfócitos $\mathrm{CD}^{+}, \mathrm{CD}^{+} \mathrm{CD}^{+} \mathrm{e}$ $\mathrm{CD}^{+}{ }^{+} \mathrm{CD} 8^{+}$e interleucina- 2 e intérferon- $\gamma$.

Óleos essenciais também podem ser usados na nutrição como método combate aos efeitos deletérios causados por micotoxinas. A piperina, componente do óleo essencial de pimenta negra (Piper nigrum) também vem se mostrando útil no combate aos efeitos negativos provocados por aflatoxinas. CARDOSO et al. (2011) apontam significativa redução da toxicidade hepática causada por aflatoxinas B1 e G1 (2ppm) na administração de $2,25 \mathrm{mg} \mathrm{kg}^{-1}$ de piperina na ração de frangos de corte, com redução das lesões histopatológicas decorrente da ingestão da toxina.

\section{Uso de adsorventes}

Os adsorventes são utilizados quando não há mais como se detoxificar os alimentos, sendo usados para impedir que as toxinas sejam absorvidas pelo trato gastrintestinal, com conseqüente diminuição dos efeitos deletérios no organismo. Um caminho para o problema tem sido usar materiais absortivos não-nutritivos na alimentação objetivando-se redução na absorção pelo trato gastrointestinal (KUBENA et al., 1990b).

Aluminosilicatos de sódio e cálcio hidratados (HSCAS)

A utilização de HSCAS em dietas de aves domésticas tem mostrado resultados satisfatórios, pela sua capacidade em adsorver toxinas, tornandoas menos disponíveis para absorção pelo trato gastrointestinal. O HSCAS na concentração de $0,5 \%$ na dieta diminuiu significativamente os efeitos adversos no peso corporal e nas principais mudanças hepáticas causados por 3,5ppm e 7,5ppm de aflatoxina B1 em frangos de corte e em poedeiras comerciais, respectivamente (KUBENA et al., 1990a; 1990b).

A eficácia dos HSCAS como adsorvente também foi comprovada por DENLI et al. (2009). Observou-se que a adição de 2 e $5 \mathrm{~g}$ de HSCAS kg-1 de ração foi capaz de diminuir significativamente $(\mathrm{P}<0,05)$ os efeitos inibitórios da Aflatoxina B1 (1ppm) sobre o desempenho zootécnico. A redução de efeitos deletérios em frangos também foi mostrada 
por KUBENA et al. (1998), uma vez que a adição de 0,25 e $0,375 \%$ de HSCAS a dietas experimentalmente contaminadas com 5 ou 8ppm de aflatoxinas $(79 \%$ $\mathrm{AFB} 1+16 \%$ AFG1+4\% AFG2+1\% AFG1) foi capaz de melhorar o ganho de peso, o consumo de ração, a conversão alimentar e a mortalidade $(\mathrm{P}<0,05)$. LEDOUX et al. (1999) também obtiveram ótimos resultados com o uso de HSCAS em dieta contaminada com 4ppm de aflatoxina B1. A adição de 1\% de HSCAS preveniu completamente a redução de desempenho zootécnico, as mudanças nos pesos dos órgãos e na bioquímica sérica, além da severidade das alterações histopatológicas de fígado e rins.

ZHAO et al. (2010) também evidenciaram o efeito positivo dos HSCAS na alimentação de frangos de corte. Tanto $0,1 \%$ quanto $0,2 \%$ de HSCAS adicionados à alimentação foram capazes de reduzir em mais de $90 \%$ os efeitos deletérios de 1ppm de AFB1 sob o desempenho zootécnico e peso de fígado $(\mathrm{P}<0,05)$.

\section{CONCLUSÃO}

A literatura mostra diversas técnicas passíveis de serem usadas como métodos auxiliares à prevenção, ou diminuição, dos prejuízos causados pelas aflatoxinas. Os adsorventes mostram-se como a alternativa mais segura e eficaz para diminuir e até evitar os efeitos deletérios causados às aves pelas aflatoxinas. À exceção dos adsorventes, embora a maioria das demais técnicas sejam eficazes, há de se observar possíveis alterações de palatabilidade e nutricional, além da produção de compostos tóxicos. Há de se considerar o nível inicial de contaminação e o tipo de técnica a ser empregada, afim de se aplicar a mais apropriada. Além disso, devese atentar para a correta destinação dos compostos resultantes da detoxificação e para a necessidade de plantas de processamento especiais; além do custo de emprego de cada método.

\section{INFORME VERBAL}

LEDOUX, D.R. Approaches for reducing the toxic effects of mycotoxins in poultry. Aula ministrada para Programa de Pós-Graduação VNP/FMVZ/USP. Pirassununga-SP. 2012. Contato: ledouxd@missouri.edu.

\section{REFERÊNCIAS}

ALEXANDRE, F.A. et al. Avaliação da eficiência da luz ultravioleta na esterilização de embalagens plásticas. Ciência e Agrotecnologia [on line], v.32, n.5, p.1524-1530, 2008. Disponível em: <http://www. scielo.br/pdf/cagro/v32n5/25.pdf>. Acesso em: maio, 2013.

Al-HILLI, A.L.; SMITH, J.E. Influence of propionic acid on growth and aflatoxin production by Aspergillus flavus in liquid submerged and solid substrate conditions. Journal of Environmental Pathology, Toxicology and Oncology, v.11, n.2, p.57-60, 1992. Disponível em: <http://www.ncbi.nlm.nih.gov/ pubmed?term=alHilli\%20AL\%5BAuthor\%5D\&cauthor=true \&ca uthor_uid=1573566>. Acesso em: maio, 2013.

AL-SOHAIBANI, S. et al. Xerophilic aflatoxigenic black tea fungi and their inhibition by Elettaria cardamomum and Syzygium aromaticum. Saudi Journal of Biological Sciences, v.18, p.387-394, 2011. Disponível em: <http://ac.els-cdn.com/ S1319562X11000465/1-s2.0-S1319562X11000465-main.pdf? tid=6e252822-1 cb2-11e3-ba4d-00000aacb361\&acdnat $=13791040$ 22 5aff2ab71e9dccda97c27de4d3ccc7d5 >. Acesso em: set. 2013. doi: 10.1016/j.sjbs.2011.06.005.

AQUINO, S. et al. Evaluation of viability of Aspergillus flavus and aflatoxins degradation in irradiated sample of maize. Brazilian Journal of Microbiology, v.36, n.4, p.352-356, 2005. Disponível em: $<$ http://www.scielo.br/pdf/bjm/v36n4/v36n4a09.pdf $>$. Acesso em: maio, 2013.

BASARAN, P.; ÜMRAN, Ü. Microwave irradiation of hazelnuts for the control of aflatoxin producing Aspergillus parasiticus. Innovative Food Science and Emerging Technologies, v.11, n.1, p.113-117, 2010. Disponível em: <http://dx.doi.org/10.1016/j. ifset.2009.08.010>. Acesso em: maio, 2013.

BATA, A.; LÁSZTITY, R. Detoxification of mycotoxincontaminated food and feeds by microorganisms. Trends in Food Science and Technology, v.10, n.6-7, p.223-228, 1999. Disponível em: <http://www.sciencedirect.com/science/article/pii/ S0924224499000503>. Acesso em: abr. 2013.

CAMARGO, A.C. et al. Efeitos da radiação gama na cor, capacidade antioxidante e perfil em ácidos-graxos de amendoim (Arachis hypogaea L.). Ciência e Tecnologia de Alimentos, v.31, n.1, p.11-15, 2011. Disponível em: <http://www.scielo.br/pdf/cta/ v31n1/02.pdf>. Acesso em: abr. 2013.

CARDOSO, V.S. et al. Efficacy of piperine in reducing the effects of aflatoxin intoxication in broiler chickens: a preliminary report. Arquivos Brasileiros de Medicina Veterinária e Zootecnia, v.63, n.2, p.495-448, 2011. Disponível em: <http://www.scielo.br/ pdf/abmvz/v63n2/31.pdf $>$. Acesso em: set. 2013.

CHEN, K. et al. Protective role of sodium selenite on histopathological lesions, decreased T-cell subsets and increased apoptosis of thymus in broilers intoxicated with aflatoxin B1. Food and Chemical Toxicology, v.59, p.446-454, 2013. Disponível em: <http://ac.els-cdn.com/S0278691513004092/1s2.0-S0278691513004092-main.pdf?_tid=41 eeaffc-1d44-11e38113-00000aacb362\&acdnat $=1379166658 \quad 0 \mathrm{c} 848 \mathrm{a} 9 \mathrm{a} 53999 \mathrm{~b}$ b4f190db0bacf8b9c3>. Acesso em: set. 2013. doi: 10.1016/j. fct.2013.06.032.

CHEN, K. et al. Effects of sodium selenite on the decreased percentage of $\mathrm{T}$ cell subsets, contents of serum IL-2 and IFN- $\gamma$ induced by aflatoxin B1 in broilers. Research in Veterinary Science, v.95, p.143-145, 2013. Disponível em: <http://ac.elscdn.com/S0034528813000738/1-s2.0-S0034528813000738-main. pdf? tid=a419056c-1d47-11e3-a9f2-00000aab0f02\&acdnat=1379 168110 4eb147f19edf966a9716c026843dbe9b>. Acesso em: set. 2013. doi:10.1016/j.rvsc.2013.02.019.

CHINAPHUTI,A.;AUKKASARAKUL,S. InhibitionofAspergillus flavus growth and aflatoxin production using herbal extracts. 
Journal of Biotechnology, v.136, p.751-759, 2008. (Resumo). Disponível em: <http://ac.els-cdn.com/S016816560801938X/1s2.0-S $016816560801938 \mathrm{X}-\mathrm{main} . \mathrm{pdf}$ ?tid=3 fee $22 \mathrm{e} 6$ $1 \mathrm{c} 9 \mathrm{f}-11 \mathrm{e} 3-8 \mathrm{db} 7-00000 \mathrm{aab} 0 \mathrm{f} 6 \mathrm{c} \& \mathrm{acdnat}=1379095785$ eba6dc1836dd098e0af9dd7cc7fb025c>. Acesso em: set. 2013. doi: 10.1016/j.jbiotec.2008.07.1672.

DENLI, M. et al. Effects of a dietary AflaDetox on performance, serum biochemistry, histopathological changes, and aflatoxin residues in broilers exposed to aflatoxin B1. Poultry Science, v.88, n.7, p. 14441451, 2009. Disponível em: < http://ps.fass.org/content/88/7/1444. full.pdf+html $>$. Acesso em: abr. 2013. doi:10.3382/ps.2008-00341.

GIORDANO, B.N.E. Efeito do ozônio sobre a micoflora e aflatoxinas durante a armazenagem de castanha-do-Brasil com casca (Bertholletia excelsea H.B.K). 2009. 193f. Dissertação (Mestrado em Ciência dos Alimentos) - Centro de Ciências Agrárias/Universidade Federal de Santa Catarina, SC.

GIORDANO, B.N.E. et al. Susceptibility of the in-shell Brazil nut microflora and aflatoxin contamination to ozone gás treatment during storage. Journal of Agricultural Science [on line], v.4, n.8, 2012. Disponível em: <http://www.ccsenet.org/journal/index. php/jas/article/view/14510>. Acesso em: maio, 2013.

GOWDA, N.K.S. et al. Efficacy of heat treatment and sun drying of aflatoxin-contaminated feed for reducing the harmful biological effects in sheep. Animal Feed Science and Technology, v.133, n.1-2,p.167-175, 2007. Disponível em: <http://www.sciencedirect. com/science?_ob=ArticleListURL\&_method=list\&_ ArticleListID $=269550983 \&$ \&_sort $=r \&$ \& $\mathrm{st}=13 \& \mathrm{view}=\mathrm{c} \&$ _ 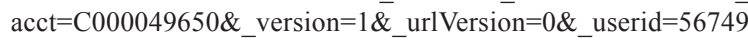 $31 \& \mathrm{md} 5=875 \mathrm{ec} 8 \mathrm{bc} 2 \mathrm{e} 1 \mathrm{c} 6 \mathrm{e} 2 \mathrm{dff5} 647 \mathrm{c} 684 \mathrm{adeb} 37 \&$ searchtype $=\mathrm{a}>$. Acesso em: abr. 2013.

HAMEED, H.G. Extrusion and chemical treatments for destruction of aflatoxin in naturally-contaminated corn. 1993. 105f. Tese (Doutorado em Ciências Nutricionais) - Comitê de Ciências Nutricionais/Universidade do Arizona.

HE, J. et al. Effects of vitamin E and selenium yeast on growth performance and immune function in ducks fed maize naturally contaminated with aflatoxin B1. Livestock Science, v.152, p.200-207, 2013. Disponível em: <http://ac.els-cdn.com/ S1871141313000255/1-s2.0-S1871141313000255-main.pdf? tid=9474c6f8-1d3f-11e3-9a6c-00000aab0f6c\&acdnat=1379164646 d6e36bd6beaaee36a418c4a35ba7e1b4>. Acesso em: set. 2013. doi: 10.1016/j.livsci.2012.12.018.

HWANG, J-H.; LEE, K-G. Reduction of aflatoxin B1 contamination on wheat by various cooking treatments. Food Chemistry, v.98, n.1, p.7175, 2006. Disponível em: <http://www.sciencedirect.com/science? $\mathrm{ob}=$ ArticleListURL\&_method=list\&_ArticleListID $=-269559790 \&$ sort $=$ r\&_st $=13 \&$ view $=$ c\&_acct $=\overline{\mathrm{C}} 000049650 \&$ \&version $=1 \&$ _ urlVersion $=0 \&$ userid $=5674931 \& \mathrm{md} 5=\mathrm{b} 213 \mathrm{dfe} 48 \mathrm{f} 5647027 \mathrm{c} 7 \mathrm{a} 5 \mathrm{ad} 1$ 39ae3470\&searchtype $=a>$. Acesso em: abr. 2013.

JEWERS, K. Mycotoxins and their effect on poultry production. In: Sauveur, B. L'aviculture en Méditerranée. Montpellier: CIHEAM (Centre International de Hautes Etudes Agronomiques Méditerranéennes), 1990. p.195-202. (Options Méditerranéennes: Série A. Seminaires Méditerrannéens; n. 07). Disponível em: $<$ http:// om.ciheam.org/om/pdf/a07/CI901593.pdf>. Acesso em: abr. 2013.

KELLS, S.A. et al. Efficacy and fumigation characteristics of ozone in stored maize. Journal of Stored Products Research, v.37, n.4, p.371-382, 2001. Disponível em: <http://www.sciencedirect. com/science? ob=ArticleListURL\& method=list \& ArticleListID $=269564061 \&$ \& sort $=$ r\&_st $=13 \&$ view $=c \&$ _ acct $=$ C000049650\&_version $=1 \&$ _urlVersion $=0 \&$ _userid $=56749 \overline{3}$

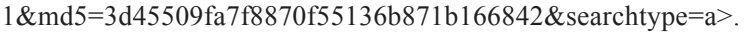
Acesso em: maio, 2013.

KUBENA, L.F. et al. Efficacy of a hydrated sodium calcium aluminosilicate to reduce the toxicity of aflatoxin and T-2 toxin. Poultry Science, v.69, n.1, p.1078-1086, 1990a. Disponível em: $<$ http://ps.fass.org/content/69/7.toc $>$. Acesso em: maio, 2013. doi: $10.3382 /$ ps.0691078.

KUBENA, L.F. et al. Diminution of aflatoxicosis in growing chickens by the dietary addition of a hidrated sodium calcium aluminosilicate. Poultry Science, v.69, p.727-735, $1990 \mathrm{~b}$. Disponível em: <http://ps.fass.org/content/69/5.toc $>$. Acesso em: maio, 2013. doi:_10.3382/ps.0690727.

KUBENA, J. F. et al. Effects of a hydrated sodium calcium aluminosilicate $\left(\mathrm{T}^{-B_{i n d}{ }^{\circledR}}\right)$ on mycotoxicosis in young broiler chickens. Poultry Science, v.77, n.10, p.1502-1509, 1998. Disponível em: $<$ http://ps.fass.org/content/77/10.toc $>$. Acesso em: maio, 2013.

LEDOUX, D.R. et al. Efficacy of a hydrated sodium calcium aluminosilicate to ameliorate the toxic effects of aflatoxin in broilers chicks. Poutry Science, v.78, n.2, p.204-210, 1999. Disponível em: <http://ps.fass.org/content/78/2.toc $>$. Acesso em: maio 2013.

LEESON, S. et al. Poultry metabolic disorders and mycotoxins. Guelph: University Books, 1995. 352p.

McKENZIE, K.S. et al. Oxidative degradation and detoxification of mycotoxins using a novel source of ozone. Food and Chemical Toxicology, v.35, n.8, p.807-820, 1997. Disponível em: <http:// www.sciencedirect.com/science?_ob=ArticleListURL\&_ method $=$ list\&_ArticleListID $=269570505 \&$ \& sort $=$ r\&_ $\mathrm{st}=13 \& \mathrm{view}=\mathrm{c} \&$ _ac $\mathrm{ct}=\mathrm{C} 000049650 \&$ \& version $=1 \&$ _ urlVersion $=0 \&$ userid $=5674931 \& \mathrm{md} 5=255440593 \mathrm{e} 46311 \mathrm{ec} 729 \overline{8}$ 950bf6fcd77\&searchtype=a>. Acesso em: abr. 2013.

McKENZIE, K.S. et al. Aflatoxicosis in turkey poults is prevented by treatment of naturally contaminated corn with ozone generated by electrolysis. Poultry Science, v.77, n.8, p.1094-1102, 1998. Disponível em: <http://ps.fass.org/content/77/8.toc $>$. Acesso em: maio 2013.

NORRED, W.P. Fumonisins - mycotoxins produced by Fusarium moniliforme. Journal of Toxicology, Environment and Health, v.38, n.3, p.309-328, 1993. Disponível em: <http://www. tandfonline.com/doi/pdf/10.1080/15287399309531720>. Acesso em: maio, 2013. doi: 10.1080/15287399309531720.

OSWEILER, G.D. Mycotoxins and livestock: What role do fungal toxins play in illness and production losses? Veterinary Medicine, v.85, n.1, p.89-94, 1990.

PIVA, G. et al. Detoxification methods of aflatoxins: a review. Nutrition Research, v.15, n.5, p.767-776, 1995. Disponível em: $<$ http://www.sciencedirect.com/science?_ob=ArticleListURL\&_ method $=$ list\&_ArticleListID $=-269620606 \&$ \& sort $=$ r\&_ $\mathrm{st}=13 \& \mathrm{view}=\mathrm{c} \&$ _ac $\mathrm{ct}=\mathrm{C} 000049650 \&$ \&version $=1 \&$ _ urlVersion $=0 \&$ userid $=5674931 \& \mathrm{md} 5=6718453 \mathrm{e} 1 \mathrm{f} 33 \mathrm{bff} 55 \mathrm{a} 2 \mathrm{cfff}$ 645038163\&searchtype $=a>$. Acesso em: maio, 2013. 
PIER, A.C. et al. Mycotoxins as a veterinary problem. In: RODRICKS, J.V. et al. Mycotoxins in human and animal health. Illinois: Pathox Publishers, 1973. p.745-750.

REDDY, K.R.N. et al. Potential of botanicals and biocontrol agents on growth and aflatoxin production by Aspergillus flavus infecting rice grains. Food Control, v.20, p.173-178, 2009. Disponível em: <http://ac.els-cdn.com/S0956713508000996/1s2.0-S0956713508000996-main.pdf? tid=28 edbb34 $1 \mathrm{cad}-11 \mathrm{e} 3-\mathrm{b} 47 \mathrm{c}-00000 \mathrm{aacb} 361$ \&acdnat $=1379101759$ c211a9bfae1f83e221f4337e97105c09>. Acesso em: set. 2013. doi: 10.1016/j.foodcont.2008.03.009.

RUSTOM, I.Y.S. Aflatoxin in food and feed: occurrence, legislation and inactivation by physical methods. Food Chemistry, v.59, n.1, p.57-67, 1997. Disponível em: <http://www.sciencedirect. com/science? ob=ArticleListURL\& method=list\& ArticleListID $=-269630691 \&$ sort $=r \&$ st $=13 \&$ view $=c \&$ acct $=$ C000049650\&_version $=1 \&$ _urlVersion $=0 \&$ userid $=5674 \overline{9}$ $31 \&$ md5 $=0 \mathrm{a} 35 \mathrm{f} 470 \mathrm{c} 4 \mathrm{fea} 3 \mathrm{a} 5 \mathrm{bab} 7116365 \mathrm{~d} 1773 \mathrm{e} \&$ searchtype $=\mathrm{a}>$. Acesso em: abr. 2013.

SAALIA, F.K.; PHILLIPS, R.D. Degradation of aflatoxins by extrusion cooking: effects on nutritional quality of extrudates. LWT - Food Science and Technology, v.44, n.6, p.1496-
1501, 2011. Disponível em: <http://www.sciencedirect. com/science? ob=ArticleListURL\& method=list \& ArticleListID $=-269635605 \&$ \& sort $=r \&$ \& $\mathrm{st}=13 \& \mathrm{view}=\mathrm{c} \&$ acct $=\mathrm{C} 000049650 \&$ version $=1 \&$ urlVersion $=0 \&$ userid $=5674 \overline{9}$ $31 \&$ md5 $=6 \mathrm{e} 2 \mathrm{~b} 2 \mathrm{f} 16 \mathrm{a} 35 \mathrm{~cd} 6 \mathrm{e} 383 \mathrm{e} 7 \mathrm{~b} 85 \mathrm{cf02}$ c4015\&searchtype $=\mathrm{a}>$. Acesso em: maio, 2013.

SAMARJEEWA, U. et al. Detoxification of aflatoxins in foods and feeds by physical and chemical methods. Journal of Food Protection, v.53, n..06, p.489-501, 1990.

SIMAS, M.M.S. et al. Influence of gamma radiation on productivity parameters of chicken fed mycotoxin-contaminated corn. Applied Radiation and Isotopes, v.68, n.10, p.1903-1908, 2010. Disponível em: <http://www.sciencedirect.com/science?_ob=ArticleListURL\& method=1ist\&_ArticleListID $=-269648611 \&$ sort $=$ r\& $\mathrm{st}=13 \&$ view $=\mathrm{c} \&$ acct $=\mathrm{C} 000049650$ \&_version $=1 \&$ \&urlVersion $=0 \&$ userid $=5674931 \& \mathrm{md} 5=451 \mathrm{e} 4 \mathrm{c} 3 \mathrm{e} 5 \mathrm{f} 2 \mathrm{fbc} 60302421 \mathrm{bdc} 246 \mathrm{ea} 35 \&$ sear chtype $=a>$. Acesso em: abr. 2013.

ZHAO, J. et al. Comparison of hydrated sodium calcium aluminosilicate and yeast cell wall on counteracting aflatoxicosis in broiler chicks. Poultry Science, v.89, n.10, p.2147-2156, 2010. Disponível em: $<$ http://ps.fass.org/content/89/10.toc $>$. Acesso em: maio, 2013. doi:_10.3382/ps.2009-00608. 